\title{
Delphi Analysis Method and Its Application in Qualitative Prediction of Aircraft Collision Unsafe Event for Air Traffic Control
}

\author{
Xu-Sheng Gan ${ }^{1, a}$, Jing-Shun Duanmu ${ }^{2, b}$, Huan-Bin Wang ${ }^{2, c}$ \\ ${ }^{1}$ XiJing College, Xi'an, Shaanxi, 710123, China \\ ${ }^{2}$ Material Management and Safety Engineering College, Air Force Engineering \\ University, Xi'an, Shaanxi, 710051, China \\ a ganxusheng123@163.com, ${ }^{b}$ dmhz521@yahoo.com.cn, ${ }^{c}$ whb1979@163.com
}

\begin{abstract}
Keywords: Delphi analysis method; Qualitative prediction; Air traffic control; Aircraft collision unsafe event
\end{abstract}

\begin{abstract}
To predict the safety situation of air traffic control, it is inadequate to only use some quantitative prediction method, which can not take into account the knowledge and experience of the experts. We should also pay attention to the important role of some qualitative prediction method. Delphi analysis method based on the opinion and consultation of the experts is proposed to predict the unsafe events in the field of air traffic control. The example shows that Delphi analysis method can be used to solve the problem of safety prediction in air traffic control.
\end{abstract}

\section{Introduction}

The prediction of air traffic control safety is that, on the basis of a certain analysis principles, use a certain method to predict the safety potential events in air traffic control activity that may occur in the aviation unit, air ATC departments, airports, airline company etc, through the analysis for flight conflict or dangerous approach, runway incursions, low-altitude flight incidents and flight error or serious unsafe incidents, general mistakes and smaller unsafe event, predict qualitatively or quantitatively the number of air traffic control safety event, and make a correct prediction of the safety position and trend of air traffic system, propose the advices for risk control and treatment measures. It also gives a good silution method for safety management, decision-making and planning about air traffic control. The paper is only concerned about the qualitative prediction problem in air traffic control safety.

When you carry out decision-making, the judgement given by only individual expert can generate the preconception. However, the consultation given by many experts together can avoid the obscurantic viewpoint. There exist also two advantages: (1) Some members can too much come under the influence of the members with plausibility and self-confidence; (2) Some members can too much come under the influence of authority and political factor.

Intuitive prediction method, which thinks of the experts as the claim object, is a qualitative prediction method that relies on the expert knowledge and experience. It is used for social development prediction, macro economic prediction, development prediction of science and technology, military equipment accident prediction, air traffic control safety prediction and so on, its accuracy depends on expert knowledge breadth, depth and experience. The experts mainly refer to the personnel who has the specialized knowledge and expertise in a certain field or a certain prediction problem. This kind of methods mainly include 3 sorts: Delphi analysis method, Scenario prediction method and Brain storm method etc.. Originally, Delphi analysis method is composed of 3 elements including: Structural information flow; Feedback result for participator; Nameless participators.

Accordingly, Delphi analysis method is introduced in the prediction of unsafe events such as flight conflict or dangerous approach, runway incursions, low-altitude flight incidents and so on in the field of air traffic control. First we introduce the origin, development, application, common principle of Delphi analysis method; and then we give the implementation flow of Delphi analysis method, finally; 
we use a acual example that a certain airline company carry out the prediction of aircraft collison unsafe events for validation.

\section{Delphi analysis method}

Delphi analysis method is also called the expert investigation method, expert advice method or expert evaluation method. It obtains the information from the experts, and relies on expert knowledge and experience, or on the basis of system procedures, through judgment, evaluation and prediction of the experts investigation for the problem, by an anonymous comment way, namely the team members do not discuss each other, only have the relationship with investigators, without the occurrence of transverse contact, in order to repeatedly fill in the questionnaire, gather the consensus in questionnaire were and collect the views of all parties, it is a method to deal with complex task difficult problem.

In 1964, USA Land Company first introduces Delphi analysis method to use in technology prediction. It is an intuitive prediction method developed on the basis of expert individual judgment and expert meeting method, especially suitable for long-term prediction under objective data or data lack case, technology prediction that other method is difficult to carry out.

Delphi analysis method as an important prediction method, has be applied in the technology prediction, economic prediction, military prediction, population prediction and so on, at the same time, its theory has also been developed and improved constantly, generating many innovative Delphy method. Due to the different application goals, Delphi analysis method has evolved into many different types, in addition to the classical Delphi and large-scale Delphi investigation, as well as cascaded Delphi method, ladder type Delphi method, decision Delphi method etc.. Based on large-scale Delphi method, the market Delphi method was put forward. The market Delphi method is a new kind of Delphi method, is to further innovation based on large-scale Delphi method. In addition to the above-mentioned anonymity, feedback, convergence and statistical characteristics of classical Delphi method, and multiple experts involved, wide survey scope, less round number, combining trend prediction and goal control characteristics of large-scale Delphi method, it also has its own remarkable characteristics, and represent in the collection candidate technology list, design market Delphi questionnaire, experts selection, emphasis on the status of target control paradigm in the technology foreseeing. Delphi analysis method is a cyclic process of many interaction with experts in "expert opinion formation, statistical feedback, opinion adjustment" form, each interaction is called a round, the work are not the same in each round.

\section{Implementation flow of Delphi analysis method}

Implement flow of Delphi analysis method is shown in Fig. 1

The implementation steps of Delphi analysis method is as follows:

1. Constitute the expert group. According to the knowledge scope that the subject requires, determine the experts. The number of experts can be determined according to the size and width of prediction task, generally not more than 20 people.

2. Propose the problem to be predicted and relevant requirements to all the experts, together with all background material on this problem, at the same time, please experts also propse to need what material. Then the experts give a written reply.

3. Each expert makes their own prediction opinions according to the material which they have received, and explain how you use these materials and gives the prediction value.

4. Collect the first judgment opinion of the experts and list the chart, in order to carry out thr comparison, and then distribute to the experts, let the experts to compare yourself with different opinion of other people, modify their opinions and judgments. You can also reorganize the opinion of the expert, or please other experts with higher identity to comment, then hand these opinions out the experts for modification opinions after the reference.

5. Collect the amended opinion of all experts, gather, once again distribute to the experts, in order to make the second revision. Round by round gathering opinion of the experts and feedback 
information for the experts is a major part of Delphi analysis method. In general, Collecting the opinions and information feedback has to go through the three or four rounds. When carry out information feedback to the expert, give only a variety of opinion, but does not indicate the expert's specific name of each opinion. This process is repeated, until every experts no longer change their opinions so far.

6. Treat the opinions of experts comprehensively. Delphi analysis method has both connections and differences with the expert meeting method that commonly include: convening a meeting of experts, collective discussion, reaching consensus prediction opinions. Delphi analysis method can play the advantages of expert meeting method: (1) It can give full play to the role of all experts, benefit by mutual discussion with high accuracy; (2) It can express disagreement point of expert opinion, take each of long, avoid various short. At the same time, Delphi analysis method also can overcome the shortcomings of expert meeting method: (1) The authoritative views influence the opinions of others; (2) Because of the sensibilities, some experts are unwilling to express the different opinions from others; (3) Motivated by the pride, some experts are not willing to modify their original opinion that is not comprehensive. The main drawback of Delphi analysis method is a complex process with long time.

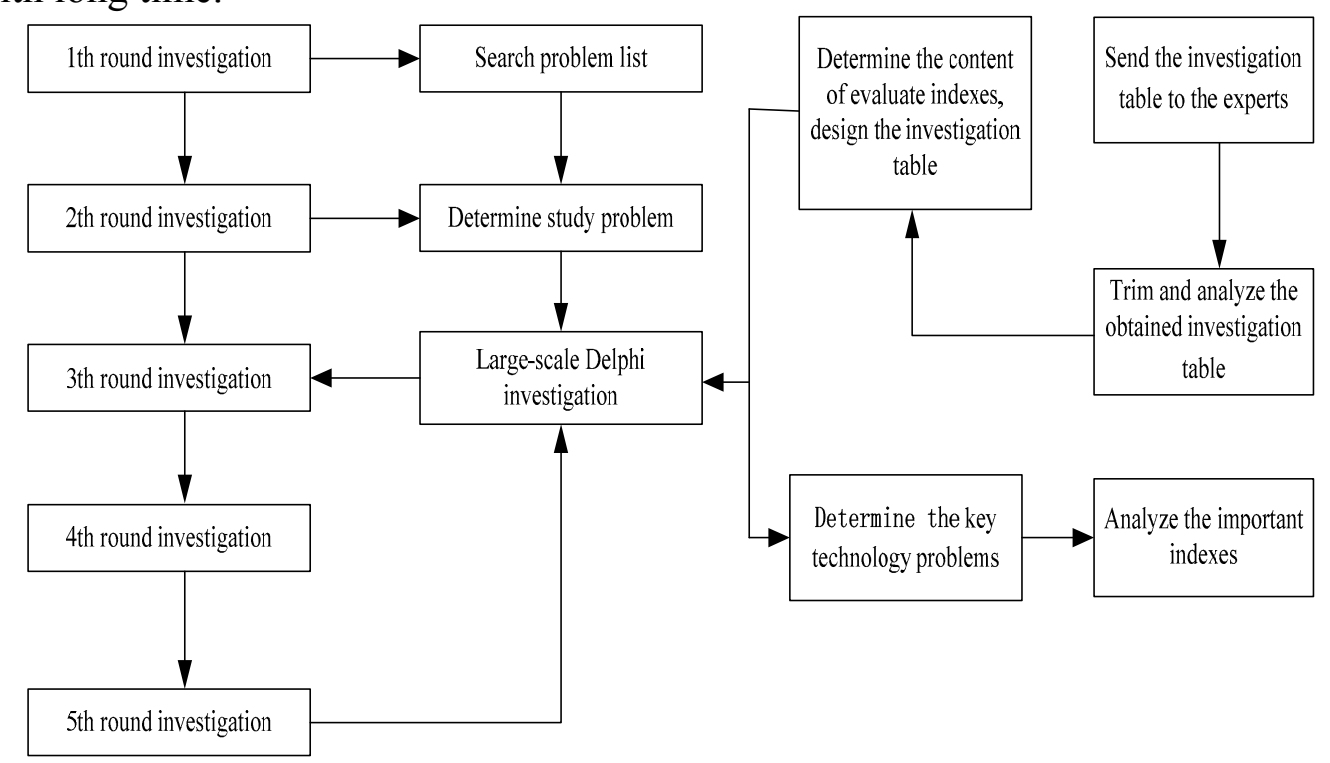

Fig. 1 Implementation flow of Delphi analysis method

\section{Actual example}

At the end of 2007, a certain airline company retains 10 experts to predict the aircraft collision unsafe events of aviation transportation in 2008. The aircraft collison unsafe events number from 2001 to 2007 is shown in Table 1. The experts need to know the relevant background information including: the quality of personnel, aircraft status, supervision mechanism, rules and regulations, prevention and control technology, the investment of funds etc.. Table 2 shows 3-th prediction opinion table of 2008 year given by 10 experts.

Table 1 Aircraft collison unsafe events from 2001 to 2007

\begin{tabular}{cccccccc}
\hline Year & 2001 & 2002 & 2003 & 2004 & 2005 & 2006 & 2007 \\
\hline Unsafe events number & 107 & 102 & 94 & 85 & 84 & 77 & 75 \\
\hline
\end{tabular}

From the table we can see that, when the experts deliver 2th round prediction opinion, most experts modify 1 th round prediction opinion, only No. 5 expert insists 1 th round of opinion. When the experts deliver 3 th round prediction opinion, only No. 2 and 8 experts insist 2 th round of the opinion. After 3 rounds of consultation, the prediction value of the experts narrow the gap gradually: 1 th round of consultation, the difference between maximum prediction value 86 of the experts and minimum 
prediction value 54 is 32 ; In 2 th round of consultation, the difference between maximum prediction value 82 of the experts and minimum prediction value 62 is 20 ; In 2 th round of consultation, the maximum prediction value of the experts is 76 , the minimum prediction value of the experts is 67 , the difference is 9 .

Table 23 -th prediction opinion table of 2008 year given by 10 experts

\begin{tabular}{ccccccccccc}
\hline Expert number & 1 & 2 & 3 & 4 & 5 & 6 & 7 & 8 & 9 & 10 \\
\hline 1th round & 72 & 70 & 74 & 54 & 62 & 78 & 73 & 64 & 86 & 71 \\
2th round & 68 & 73 & 71 & 69 & 62 & 75 & 74 & 72 & 82 & 78 \\
3th round & 70 & 73 & 75 & 68 & 69 & 71 & 76 & 72 & 74 & 67 \\
\hline
\end{tabular}

Use the average method to determine the final prediction value

$(70+73+75+68+69+71+76+72+74+67) / 10=71.5 \approx 72$

The prediction results of airline aircraft collison unsafe event number in 2008 is 72 .

Because of the limitation of paper length, we can not express the process of opinion transformation of 10 experts completely. In fact, when the experts predict the unsafe events, there exist a very complex psychology change. They also need to synthesize various information from different sources to make themselves judgements. In addition, it can be seen from the realization process of Delphi analysis method that the method can be associated with the practical safty situation of air traffic control.

\section{Conclusions}

Delphi analysis method, a qualitative prediction method, has be used in so many safety demain, and it has been proven that it is a more effective prediction approach. In the paper, Delphi analysis method is introduced in the field of air traffic control to solve the problem on the prediction of unsafe events in order to carry out the safety management and decision-making effectively. The actual example on the aircraft collison unsafe events number for a certain airline company shows that, Delphi analysis method has a strict logistic reasoning relation. Its application process is very fit for thinking mode of the human. It is feasible to use Delphi analysis method to predict the safety trend of air traffic control system.

\section{References}

[1] Q. M. Zhang, G. J. Zhang, w. Wang, et al. Research on prediction of technology based on Delphi survey method and route chart technology-taking technological development prediction of Taiyuan the twelfth five development plan for an example. Sci-Tech Innovation \& productivity, $10,(2010), 75-79$

[2] W. T. Liu,H. Gu, C. H. Li. Expert evaluation method based on Delphi method. Computer Engineering, 37, (2011), 189-191

[3] J. Tian, P. Z. Zhang, K. L. Wang, Y. L. Wang. The integrating model of expert's opinion based on Delphi Method. System Engineering Theory \& Practice, 1, (2004), 57-62

[4] X. J. Kuang. Study on the affecting factors of the development of drifting tourism in china based on Delphi method. Tourism Tribune, 26(6), (2011),42-46

[5] H. B. Hu, J. J. Qiu, A. M. Ma. Performance analysis of unknown mine based on Delphi Technique. Information Command Control System \& Simulation Technology, 27(2), (2005),12-14 